\title{
Inversão sexual de alevinos de tilápias do Nilo (Oreochromis niloticus) variedade Supreme, submetidos a diferentes temperaturas durante fase de diferenciação sexual
}

\author{
Sex revesal of Nile tilapia (Oreochromis niloticus) Supreme \\ variety, fingerlings submitted to different temperatures for sexual \\ differentiation phase
}

\author{
Marco Antonio Zanoni ${ }^{1 *}$; Thais Vasconcelos Leal2; Mauro Caetano Filho ${ }^{3}$; \\ Carlos Antonio Lopes de Oliveira ${ }^{4}$; Ricardo Pereira Ribeiro ${ }^{4}$
}

Resumo

Este experimento avaliou os efeitos da temperatura na inversão sexual, sobrevivência e presença de deformidades em larvas de tilapia submetidas aos seguintes tratamentos controle $\left(28^{\circ} \mathrm{C}+\right.$ ração $60 \mathrm{mg} \mathrm{kg}^{-1}$ de $17 \alpha$-metiltestosterona), $28,30,32$ e $34^{\circ} \mathrm{C}$. Os resultados demonstraram diferença estatística entre a proporção de machos, sendo que as temperaturas controle, 32 e $34^{\circ} \mathrm{C}(98 ; 91 ; 96 \%$, respectivamente) apresentaram resultados superiores aos obtidos para os tratamentos 28 e $30^{\circ} \mathrm{C}(71$ e $82 \%$ ). A sobrevivência observada nos diferentes tratamentos foram 90,74; 90,49; 88,20;85,43 e 82,16 para o controle, $28,30,32$ e $34^{\circ} \mathrm{C}$, respectivamente, sendo que houve diferença estatística entre os tratamentos 32 e $34^{\circ} \mathrm{C}$, somente quando estes foram comparados com o controle. Na análise das radiografias não foi possível observar presença de alterações morfológicas nos peixes submetidos aos diferentes tratamentos. Com este experimento, concluiu-se que as temperaturas de 32 e $34^{\circ} \mathrm{C}$ foram capazes de masculinizar larvas geneticamente fêmeas, e que não houve incremento na mortalidade e na presença de deformidades que possam ser atribuídas aos tratamentos. Essa técnica é uma alternativa para a utilização de hormônios esteroides.

Palavras-chave: Inversão sexual, sobrevivência, alterações morfológicas, proporção sexual

\begin{abstract}
This experiment evaluated the effects of temperature on sex inversion survival and presence of deformities in larvae of tilapia subjected to the following treatments control $\left(28^{\circ} \mathrm{C}+\right.$ diet $60 \mathrm{mg} \mathrm{kg}-1$ of $17 \alpha$-methyltestosterone), 28, 30, 32 and $34^{\circ} \mathrm{C}$. The results showed a statistical difference between the proportion of males, with temperatures control, 32 and $34^{\circ} \mathrm{C}(98,91,96 \%$, respectively) showed better results than those obtained for treatments 28 and $30^{\circ} \mathrm{C}$ (71 and $\left.82 \%\right)$. The survival observed in different treatments were $90.74,90.49,88.20,85.43$ and 82.16 for the control, $28,30,32$ and $34^{\circ} \mathrm{C}$, respectively, and there was statistical difference between treatments 32 and $34{ }^{\circ} \mathrm{C}$, only when these were compared with the control. In the analysis of the radiographs was not possible to observe the presence
\end{abstract}

\footnotetext{
${ }^{1}$ Prof. do Setor de Biologia, Centro de Ciências Biológicas, Universidade Estadual Norte do Paraná, UENP, Campus Luiz Meneghel, Bandeirantes, PR. E-mail: zanoni@uenp.edu.br

${ }^{2}$ Discente do curso de Ciências Biológicas, UENP, Bandeirantes, PR. E-mail: leal.bio@hotmail.com

${ }^{3}$ Biólogo responsável pela Estação de Piscicultura, UENP, Centro de Ciências Biológicas, Londrina, PR. E-mail: mcaetanofilho@ hotmail.com

${ }^{4}$ Profs. do Dept ${ }^{\circ}$ de Zootecnia, da Universidade Estadual de Maringá, UEM, Maringá, PR. E-mail: caloliveira@uem.br; rpribeiro@ uem.br

* Autor para correspondência
} 
of morphological changes in fish subjected to different treatments. With this experiment, we concluded that the temperatures of 32 and $34^{\circ} \mathrm{C}$ were able to genetically larvae masculinizing females, and that there was no increase in mortality and the presence of deformities could be attributed to treatment. This technique is an alternative to use of steroid hormones.

Key words: Sex reversal, suvirval, morphological changes, sex ratio

\section{Introdução}

O cultivo de peixes em tanques e viveiros vem tornando-se uma atividade econômica de grande importância para a produção de proteína animal. A tilápia do Nilo (Oreochromis niloticus) é uma espécie rústica e amplamente cultivada, pois se desenvolve em uma extensa faixa de condições ambientais (CARDOSO FILHO; CAMPECHE; PAULINO, 2010), possui grande versatilidade alimentar (SANTOS et al., 2009), tolera bem o estresse causado por manipulação (TSADIK; BART, 2007) e é bastante apreciada pelo mercado consumidor (SANTANA et al., 2010).

Por outro lado, as características reprodutivas da tilápia do Nilo, como alta capacidade de reprodução, maturidade sexual precoce, fecundidade relativamente elevada e desova frequente, tem gerado um dos principais problemas encontrados pelos criadores de tilápias, que é a superpopulação dentro dos viveiros de cultivo, prejudicando a taxa de crescimento dos indivíduos (POPMA; GREEN, 1990; BORGES, 2002), reduzindo a produtividade e inviabilizando a produção comercial.

O cultivo racional da tilápia tem como uma das suas prioridades, a inversão sexual de fêmeas genéticas para machos fenotípicos, os quais possuem crescimento mais rápido, e também evita problemas provenientes da reprodução e desvio de energia que seria para o crescimento (MEURER et al., 2005). O método mais comum para a criação de populações monossexo é a inversão sexual de larvas com a utilização de rações contendo hormônios esteroides sexuais sintéticos (POPMA; GREEN, 1990). Entre os hormônios pesquisados, o andrógeno sintético 17 $\alpha$-metiltestosterona tem sido bastante empregado no processo de reversão sexual, por apresentar a vantagem de ser facilmente excretado logo após o período do tratamento hormonal (POPMA; GREEN, 1990; ROTHBARD et al., 1990; CURTIS et al., 1991; PHELPS; POPMA, 2000).

A influência da temperatura na inversão do sexo de peixes foi inicialmente demonstrada em estudos sobre o peixe rei Menidia menidia, por Conover e Kynard (1981) que demonstraram um período termossensível durante a ontogênese. Estudos mais recentes (BARAS et al., 2000; WANG; TSAI, 2000; BARAS; JACOBS; MÉLARD, 2001; BARAS et al., 2002; KARAYÜCEL et al., 2003; BORGES et al., 2005; BEZAULT et al., 2007; WESSELS; HÖRSTGEN-SCHWARK, 2007; AZAZA; DHRAÏEF; KRAÏEM， 2008; ANGIENDA; AKETCH; WAINDI, 2010; WESSELS; SAMAVATI; HÖRSTGEN-SCHWARK, 2011) confirmam essa característica em diferentes espécies de tilápia, que apresentam desvios significativos na proporção de fenótipos masculinos quando submetidos a temperaturas altas na fase inicial de desenvolvimento, quando o sexo fenotípico do indivíduo ainda não se desenvolveu.

O objetivo deste trabalho foi avaliar a influência da temperatura no percentual de machos, sobrevivência e presença de deformidade, de larvas de tilápia do Nilo (O. niloticus) mantidas durante o período de diferenciação sexual em diferentes temperaturas.

\section{Material e Métodos}

O experimento foi realizado no Laboratório da Estação de Piscicultura da Universidade Estadual de Londrina (Epuel), no período de abril a maio de 2011, com duração de 28 dias. As larvas de tilápia, com 48 h pós-eclosão, utilizadas nesse experimento, foram obtidas junto à empresa Aquabel (Rolândia, 
Estado do Paraná). As 1.200 larvas transportadas em sacos plásticos foram distribuídas em 30 grupos de 40 indivíduos escolhidos ao acaso. Cada grupo foi transferido para uma unidade experimental, preparada com a utilização de garrafas plásticas com volume de $2 \mathrm{~L}$, que tiveram o fundo retirado para facilitar o manejo alimentar; a tampa foi substituída por um pedaço de tela plástica "mosquiteiro" e a região mediana foi perfurada para facilitar a troca de água com o meio externo.

De um total de 30 unidades experimentais, 24 foram montadas no interior de quatro incubadoras do tipo Woynarovich de 220 L (seis unidades experimentais/incubadora). Após um período de adaptação de $24 \mathrm{~h}$, à temperatura das incubadoras foram acrescidas em $2^{\circ} \mathrm{C}$ a cada dia, com a ajuda de aquecedores e termostatos elétricos, até atingir as temperaturas propostas para o experimento que foram $28,30,32$ e $34^{\circ} \mathrm{C}$. A ração oferecida para as larvas nesses tratamentos continha $48 \%$ de proteína bruta. Uma quinta incubadora de 220 L, foi utilizada como controle onde seis unidades experimentais foram mantidas à temperatura de $28^{\circ} \mathrm{C}$ recebendo dieta com $60 \mathrm{mg}$ de $17 \alpha$-metiltestosterona por kilograma de ração com $48 \%$ de proteína bruta. A renovação de água nas incubadoras utilizadas no experimento foi constante e ajustada à manutenção da temperatura proposta em cada uma.

O crescimento das larvas durante o experimento foi avaliado por meio de biometrias. Na fase inicial, uma amostra de 15 indivíduos foi retirada ao acaso antes das larvas serem distribuídas nas unidades experimentais. Outras amostras de cinco indivíduos de cada repetição foram tomadas a cada 14 dias (14 e 28 dias). Nas três coletas, os animais foram insensibilizados com benzocaina $\left(3 \mathrm{~g} 100 \mathrm{~mL}^{-1} \mathrm{de}\right.$ álcool), sacrificados em álcool 70, e armazenados em frascos de vidro identificados por tratamento. Posteriormente, as larvas foram então medidas com o auxílio de um paquímetro digital, secas em papel toalha e pesadas em balança analítica, a média dos pesos obtidos em cada biometria foi utilizada para calcular a curva de ganho em peso.
Ao término do $28^{\circ}$ dia de tratamento a etapa de inversão sexual foi encerada e as larvas transferidas para caixas d'água plásticas de 500 L. A temperatura, durante essa fase, foi mantida em $27^{\circ} \mathrm{C}$ com o auxílio de um aquecedor elétrico. O manejo alimentar foi realizado duas vezes ao dia, oferecendo-se ração comercial com $28 \%$ de proteína bruta, até a saciedade. A renovação da água foi constante e a cada dois dias foi realizada a sinfonagem da água, para facilitar, a remoção da matéria orgânica acumulada.

Após 60 dias de cultivo, dez indivíduos de cada repetição foram retirados aleatoriamente, insensibilizados com o auxílio de benzocaína, fixados em solução bouin e armazenados em vidros identificados. Para a análise da porcentagem de inversão sexual, os alevinos após fixação, tiveram sua cavidade abdominal aberta com o auxílio de tesoura e pinça. As suas gônadas foram retiradas e transferidas para uma lâmina, onde foram coradas com acetato-carmim, prensadas com o auxílio de uma lamínula (Squash) e avaliadas em microscopia óptica em aumento de 40 vezes.

Para a avaliação de possíveis alterações morfológicas, uma segunda amostra de dez indivíduos de cada tratamento foram insensibilizados com benzocaina e sacrificada em álcool 70, e examinadas por meio de radiografias (adaptado de ALMEIDA, 2003) em equipamento radiológico General Eletric ${ }^{\circledR}$ modelo 1000 utilizando-se filmes $\operatorname{Kodak}^{\circledR}$ com área de $18 X 24 \mathrm{~cm}$, a distância focal foi de $60 \mathrm{~cm}$ e o equipamento calibrado em $10 \mathrm{~mA}$ e $45 \mathrm{KV}$, os peixes foram distribuídos sobre o chassi radiológico na posição lateral. Para a avaliação do desvio de coluna, duas linhas foram traçadas sobre a imagem da radiografia; uma ligando a parte inicial da coluna vertebral (próximo à cabeça) à parte final da coluna e a segunda ligando a extremidade posterior da primeira linha ao ponto mais distante da curvatura da coluna. Os animais foram considerados como portador de desvio na coluna vertebral em que o ângulo formado entre as duas linhas foi superior a $15^{\circ}$. Também foi observado se houve a presença de alterações na forma dos ossos da cabeça e opérculo. 
A qualidade de água foi avaliada semanalmente para as seguintes variáveis: $\mathrm{pH}$ (potenciômetro digital), oxigênio dissolvido ( $\mathrm{mg} \mathrm{L}^{-1}$ ) (oxímetro YSI - Yellow Spring Instruments), alcalinidade $\mathrm{CaCO}_{3}$ (mg L $\mathrm{m}^{-1}$ ) e amônia $\left(\mathrm{mg} \mathrm{L}^{-1}\right)$, utilizando a metodologia de Golterman, Clymo e Ohnstad (1978).

Os dados obtidos de sobrevivência e porcentagem de machos por apresentarem a caracterização binária, em que se verifica a sobrevivência ou a morte e a ocorrência de machos ou fêmeas, foram submetidas a análises estatísticas utilizando a metodologia de modelos lineares generalizados, implementado no PROC GENMOD do sistema computacional SAS (versão 2002-2004). Para ambas as variáveis, considerou-se a distribuição binomial com função ligação logit. Ajustou-se uma regressão logística da percentagem de sobrevivência e ocorrência de machos em função das diferentes temperaturas de cultivo. Comparou-se o tratamento controle (de $28^{\circ} \mathrm{C}$ recebendo dieta com $60 \mathrm{mg}$ de $17 \alpha$-metiltestosterona por kilograma de ração) com aqueles em que as temperaturas variaram.

Para avaliar o ganho em peso dos animais submetidos aos diferentes tratamentos nesse experimento, os resultados de peso final obtidos na última biometria foram submetidos ao teste de análise de variância.

\section{Resultados e Discussão}

Os resultados obtidos das variáveis físicas e químicas da água, no interior das unidades experimentais foram: oxigênio dissolvido $\left(\mathrm{mg} \mathrm{L}^{-1}\right)$ : $4,4 \pm 0,8 ; \mathrm{pH}: 6,8 \pm 0,2$; alcalinidade total: $60 \mathrm{mg} \mathrm{L}^{-1}$ e amônia total: $<0,5 \mathrm{ppm}$. A temperatura mantevese dentro das faixas propostas para os tratamentos 28 , controle ( $28+60 \mathrm{mg}$ de $17 \alpha$-metiltestosterona), 30,32 e $34^{\circ} \mathrm{C}$ e os valores médios foram de 28,06 $\pm 0,24 ; 28,10 \pm 0,28 ; 30,60 \pm 2,33 ; 32 \pm 0,61 \mathrm{e}$ $34,44 \pm 0,26$, respectivamente. Os resultados de oxigênio dissolvido, $\mathrm{pH}$, alcalinidade total e amônia total observados nesse experimento encontram-se dentro da faixa do recomendada por Boyd (1990), Popma e Lovshin (1996), contudo a temperatura, nos tratamentos com 30,32 e $34^{\circ} \mathrm{C}$, é considerada acima da recomendada pelos autores, o que pode influenciar a qualidade do cultivo e na sobrevivência das larvas.

As curvas de ganho em peso podem ser observadas na Figura 1, e os resultados de peso médio inicial e os de peso médio final de cada tratamento e do controle podem ser observados na Tabela 1. Estes resultados são inferiores aos obtidos por Costa et al. (2011), os quais obtiveram valores de peso final após 28 dias de cultivo, que variaram de 0,23 a 0,35 g, para densidade (15 larvas/L) semelhante a deste experimento (12 larvas/L) e temperatura de $28{ }^{\circ} \mathrm{C}$. Contudo, esses pesquisadores trabalharam com suplementação alimentar das larvas com algas microscópicas (Chlorella sp), o que pode explicar os melhores resultados obtidos. 
Figura 1. Curva de ganho em peso das larvas de tilápia do Nilo mantidas em diferentes temperaturas: $28,30,32$ e $34^{\circ} \mathrm{C}$ e alimentadas com ração contendo $60 \mathrm{mg} \mathrm{kg}^{-1}$ de $17 \alpha$-metiltestosterona, por um período de 28 dias.

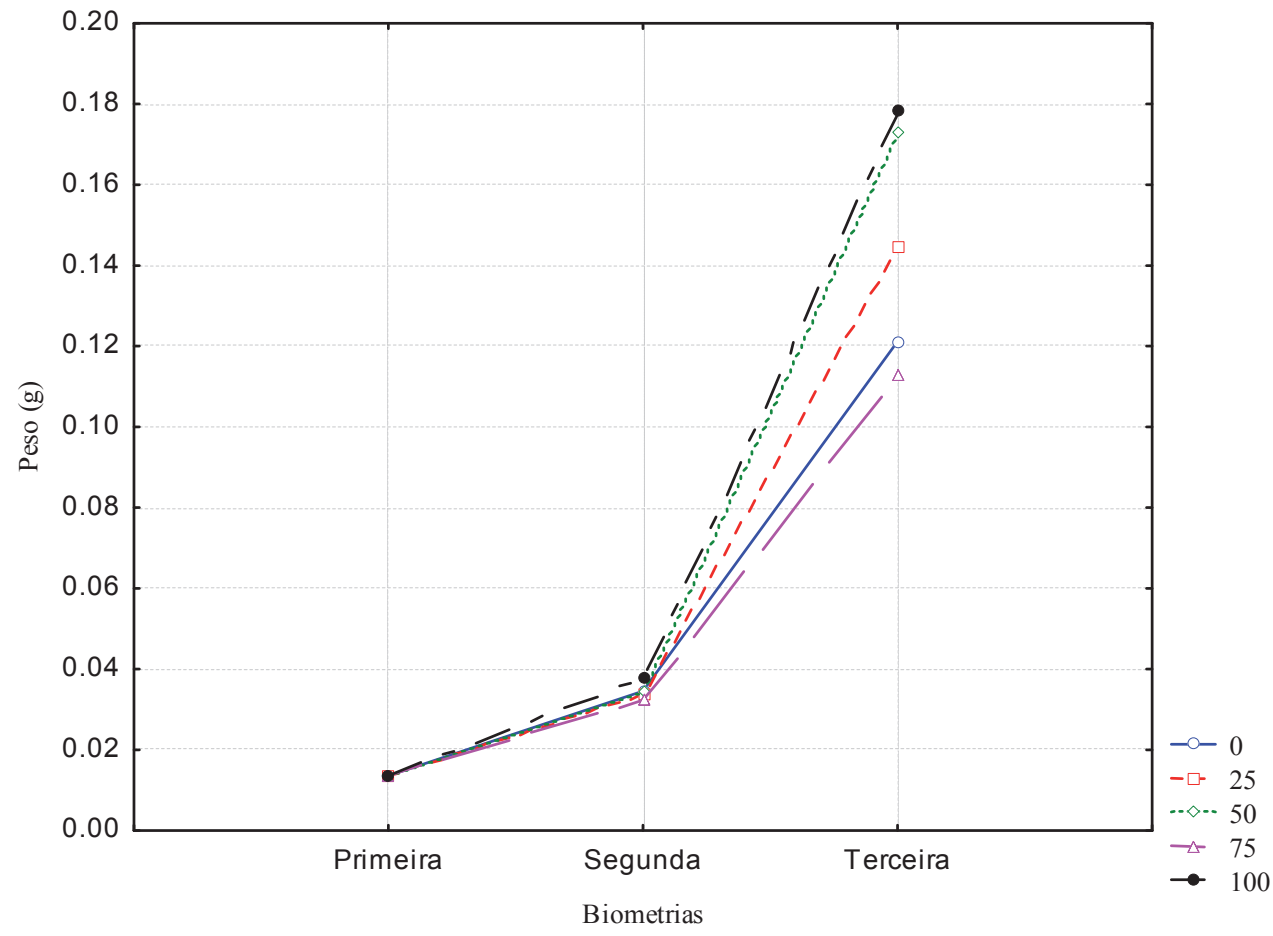

Fonte: Elaboração dos autores.

Tabela 1. Resultados obtidos do peso médio inicial, peso médio final e sobrevivência da tilápia do Nilo submetida à inversão sexual por diferentes temperaturas: controle $\left(28^{\circ} \mathrm{C}+60 \mathrm{mg}\right.$ de $17 \alpha$-metiltestosterona $), 28,30,32,34^{\circ} \mathrm{C}$.

\begin{tabular}{cccc}
\hline Temperatura ${ }^{\circ} \mathrm{C}$ & \multicolumn{3}{c}{ Variável } \\
\hline & Peso inicial $(\mathrm{g})$ & Peso Final $(\mathrm{g})$ & Sobrevivência (\%) \\
\cline { 2 - 4 } Controle & $0,012 \pm 0,005$ & $0,13 \pm 0,046^{\mathrm{a}}$ & $90,74 \pm 2,28^{\mathrm{a}}$ \\
28 & $0,012 \pm 0,005$ & $0,10 \pm 0,029^{\mathrm{a}}$ & $90,49 \pm 2,32^{\mathrm{a}}$ \\
30 & $0,012 \pm 0,005$ & $0,10 \pm 0,035^{\mathrm{a}}$ & $88,20 \pm 2,04^{\mathrm{ab}}$ \\
32 & $0,012 \pm 0,005$ & $0,09 \pm 0,0291^{\mathrm{a}}$ & $85,43 \pm 1,5^{\mathrm{ab}}$ \\
34 & $0,012 \pm 0,005$ & $0,11 \pm 0,06^{\mathrm{a}}$ & $82,16 \pm 1,63^{\mathrm{b}}$ \\
\hline
\end{tabular}

* Letras semelhantes na mesma coluna demonstram haver diferença estatística entre as médias (Tukey)

Fonte: Elaboração dos autores.

A análise estatística demonstrou não haver diferenças significativas de peso médio final nos no controle e nos diferentes tratamentos, os quais diferem dos resultados obtidos por Baras, Jacobs e Mélard (2001) que afirmaram que a exposição de larvas de tilápia do Nilo a temperaturas que podem propiciar a inversão de sexo para macho, pode diminuir significativamente as taxas de crescimento quando comparadas com temperaturas normais de cultivo. Essa redução de ganho de peso observada pelos autores é explicada, pelo fato de que os peixes reduzem o consumo ou mesmo cessam a alimentação, quando estão fora da faixa de conforto térmico para a espécie (SCHMITTOU, 1993).

$\mathrm{O}$ efeito anabólico do $17 \alpha$-metiltestosterona não foi observado neste experimento, sendo que os 
resultados em ganho em peso das larvas de tilápia não estão de acordo com os de Dias-Koberstein et al. (2007) que observaram diferenças significativas no ganho em peso de larvas alimentadas com dietas contendo este esteróide, e que foram mantidas a temperatura de $28^{\circ} \mathrm{C}$. A ausência de efeito anabólico nesse experimento pode ser explicada por Rinchard et al. (1999) que afirmam que são vários os fatores que influenciam o crescimento diferenciado dos animais alimentados com $17 \alpha$-metiltestosterona, entre os fatores citados estão: o estágio de desenvolvimento da larva, tempo de administração do hormônio, fatores dietéticos, método de aplicação do hormônio e a temperatura, a qual neste experimento variou de 28 a $34^{\circ} \mathrm{C}$.

Os valores médios de sobrevivência podem ser observados na Tabela 1. A analise de variância das médias obtidas demonstrou haver diferença estatística (1\%) entre os tratamentos e o teste de comparação de médias (Tukey), demonstrou haver similaridade entre as médias obtidas no controle e as temperaturas de 28,30 e $32^{\circ} \mathrm{C}$. Já o tratamento em que as larvas foram mantidas a temperatura de $34^{\circ} \mathrm{C}$, apresentou uma média menor de sobrevivência que os demais, mas semelhante às médias observadas na temperatura de 30 e $32^{\circ} \mathrm{C}$ e diferente das obtidas no controle e a $28^{\circ} \mathrm{C}$. Estes resultados estão de acordo aos observados em estudo realizado por Rougeot et al. (2008), onde os autores observaram que durante o período de diferenciação sexual, a sobrevivência de larvas tilápia do Nilo diminuiu drasticamente quando submetidas a temperaturas acima de $35^{\circ} \mathrm{C}$.

No entanto Desprez e Mélard (1998) não observaram diferenças significativas de sobrevivência de larvas de tilápia azul (O. aureus), quando submetidas a temperaturas de 34 e $27^{\circ} \mathrm{C}$ (controle). E segundo Wessels, Samavati e HörstgenSchwark (2011) a idade das larvas tem grande influência neste parâmetro, sendo que larvas com mais de $15 \mathrm{~h}$ pós-fertilização são mais resistentes a mortandade quando submetidas ao processo de inversão sexual utilizando temperaturas altas, do que larvas com menos de $12 \mathrm{~h}$ pós-fertilização. Essa maior resistência e sobrevivência a temperaturas altas por larvas de tilápia com mais de 15 horas de vida, não foi observada neste experimento, que utilizou larvas da variedade Supreme com mais de 48 horas de vida.

Os resultados médios de porcentagem de indivíduos machos e seus respectivos desvios padrões, dentro dos tratamentos em que foi testado diferentes temperaturas $28,30,32,34^{\circ} \mathrm{C}$ e controle podem ser observados na Figura 2, a análise de regressão logística indicou que com o aumento da temperatura houve aumento na porcentagem de machos (Figura 3). A comparação das médias obtidas pela análise estatística demonstrou não haver diferença significativa entre os tratamentos 28 e $30^{\circ} \mathrm{C}$ e entre os tratamentos 32 e $34^{\circ} \mathrm{C}$, no entanto quando as médias dos tratamentos 28 e 30 ${ }^{\circ} \mathrm{C}$ foram comparadas com os $32,34{ }^{\circ} \mathrm{C}$ e controle, há diferença estatística significativa $(\mathrm{p}<0,05)$.

Os resultados obtidos com a tilápia do Nilo variedade Supreme demonstram haver um comportamento quadrático $(\mathrm{P}<0,05)$ entre o maior número de indivíduos machos e o aumento da temperatura, com nível para melhor desempenho em temperatura de 32 e $34^{\circ} \mathrm{C}$, confirmando os resultados já obtidos por diversos autores em estudos prévios com tilápias do Nilo (BAROILLER et al., 1995; LIKONGWE et al., 1996; ABUCAY et al., 1999; BARAS; JACOBS; MÉLARD, 2001; KARAYÜCEL et al., 2003; BORGES et al., 2005; BEZAULT et al., 2007; WESSELS; HÖRSTGEN-SCHWARK, 2007; AZAZA; DHRAÏEF; KRAÏEM, 2008; ROUGEOT et al., 2008; ANGIENDA; AKETCH; WAINDI, 2010; WESSELS; SAMAVATI; HÖRSTGENSCHWARK, 2011), bem como para outras espécies de tilápias (DESPREZ; MÉLARD, 1998; BARAS et al., 2000; WANG; TSAI, 2000; BARAS et al., 2002). 
Figura 2. Valores médios, erro padrão e desvio padrão dos resultados de inversão sexual nos tratamentos realizados com diferentes temperaturas.

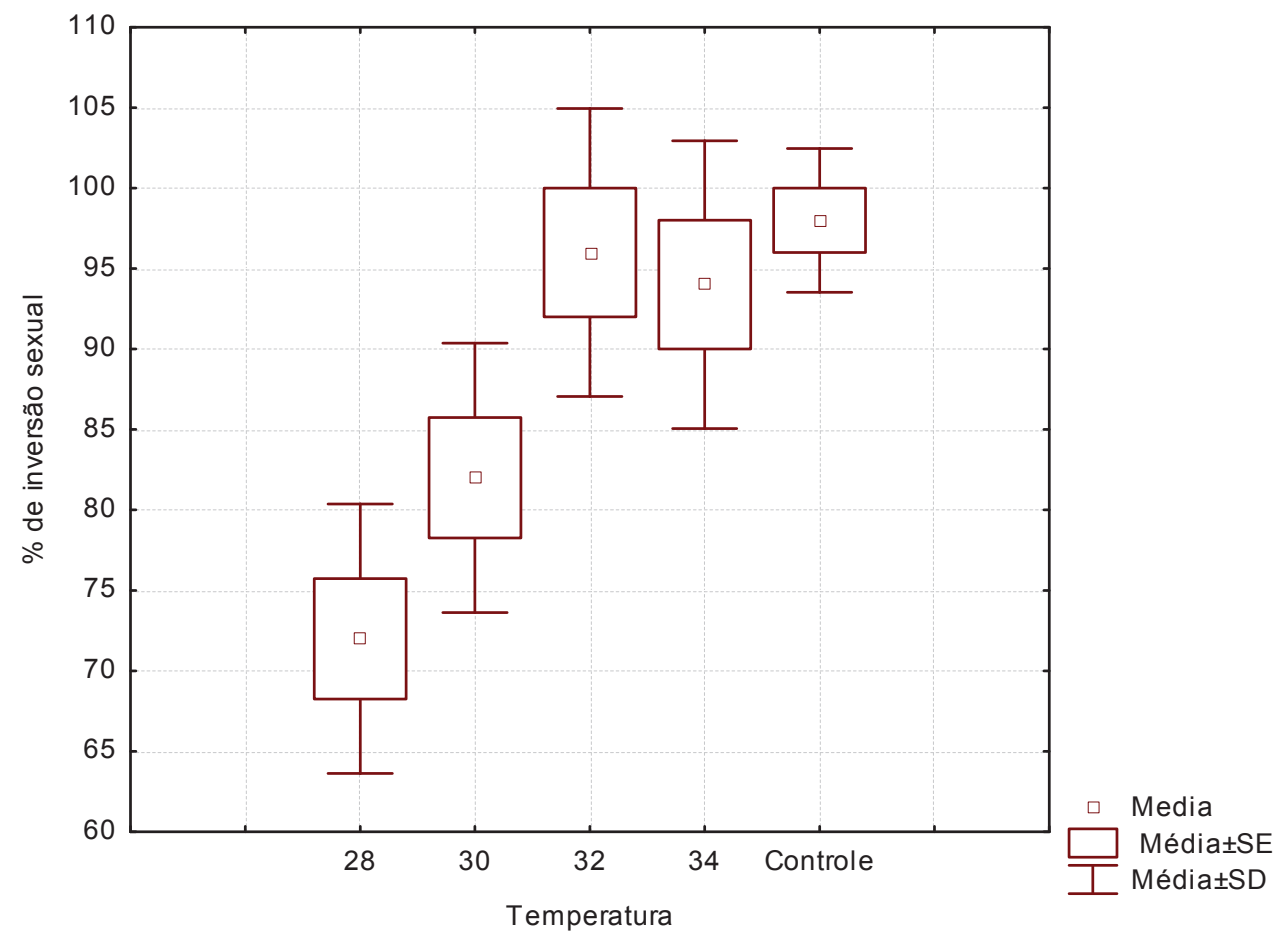

Fonte: Elaboração dos autores.

Figura 3. Reta e equação da regressão linear da inversão sexual de alevinos de tilápia do Nilo induzidos por diferentes temperaturas.

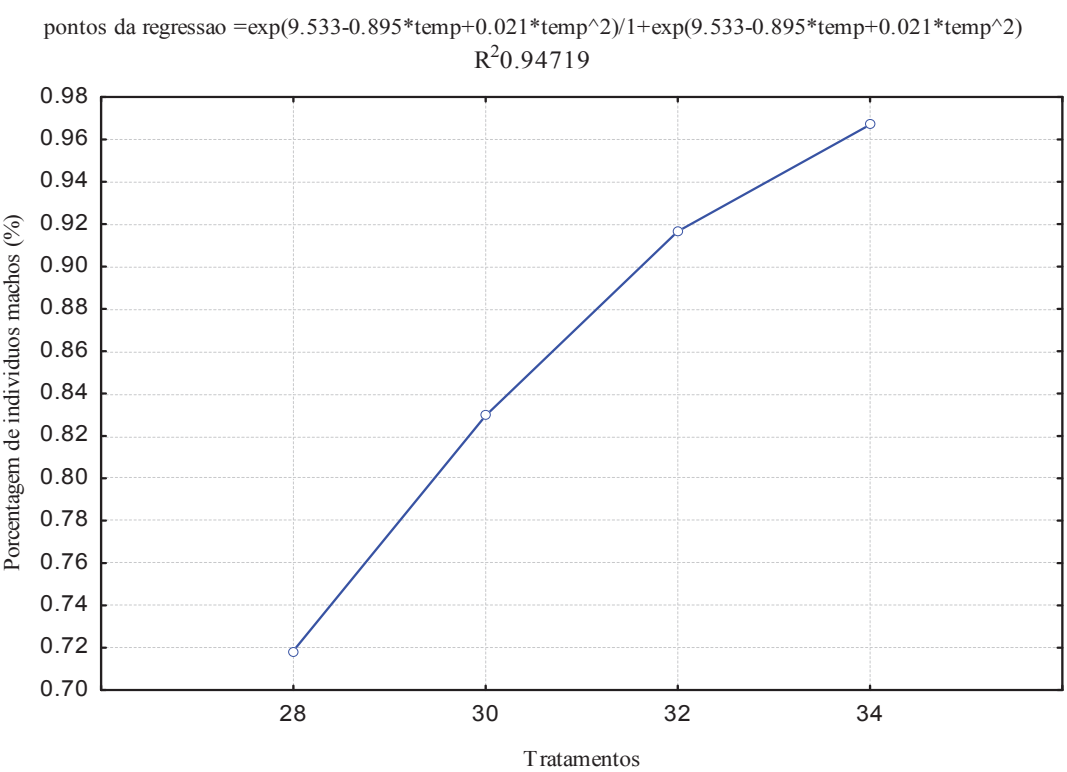

Fonte: Elaboração dos autores. 
O aumento na proporção de indivíduos machos, observado em larvas tilápia do Nilo submetidas à temperatura alta deve-se, segundo Abucay et al. (1999), provavelmente a um efeito sobre a estrutura ou a ação de um ou mais hormônios que agem na diferenciação sexual. Wang e Tsai (2000) afirmaram que a determinação do sexo em peixes é baseada na concorrência entre a $5 \alpha$-reductase e a P450 aromatase pelo substrato andrógeno no cérebro e nas gônadas indiferenciadas, sendo que as temperaturas que determinam o sexo feminino podem promover aumento na produção de receptores de aromatase e/ou estrogênio que, por sua vez, promovem o desenvolvimento do sexo feminino. Por outro lado, a determinação do sexo masculino pela temperatura, deve-se ao aumento na produção de redutase e/ou receptor de andrógeno.

Algumas variedades de tilápia, segundo Abucay et al. (1999) e Baras, Jacobs e Mélard (2001), são mais susceptíveis aos efeitos da temperatura na proporção de macho, do que outras variedades. Esses autores relataram resultados em que a razão sexual de progênies, de diferentes reprodutores, criados em $35^{\circ} \mathrm{C}$ variou entre 51 a $90 \%$. Resultados semelhantes também foram encontrados por Baroiller et al. (1995) ao relatar que reprodutores da mesma variedade poderiam resultar em progênies que seriam pouco ou muito pouco sensíveis à temperatura. Essa discrepância entre os resultados obtidos dentro de uma mesma variedade de tilápias, segundo Baras, Jacobs e Mélard (2001), pode ser atribuída à heterogeneidade das linhagens avaliadas, sendo que quanto maior heterose, maior a variação na proporção dos sexos.

Neste experimento, as porcentagens de macho obtidos nas seis repetições dos diferentes tratamentos foram uniformes, demonstrando um baixo grau de heterose. Esta afirmação é comprovada por Baroiller et al. (1995) que indicam as linhagens Chitralada e a Boauké, como possuindo maior sensibilidade aos tratamentos com temperatura. Borges et al. (2005) postularam que essas duas linhagens representam as melhores opções para o desenvolvimento da tecnologia de produção de populações monossexo macho por meio da temperatura.

Os alevinos de tilápia do Nilo escolhidos aleatoriamente para serem radiografados apresentaram tamanho médios de 51,8 \pm 11,6 mm e as radiografias (Figura 4) e demonstram não haver deformidades estruturais na coluna vertebral, costelas, ossos do crânio, mandíbula, maxila e opérculos. Diferentemente deste, experimento realizado por Wang e Tsai (2000) observaram que quando larvas de tilápia (O. mossambicus) com zero a cinco dias de idades foram expostas a temperaturas entre 28 a $32^{\circ} \mathrm{C}$, houve aumento na proporção de indivíduos que apresentaram deformidades, enquanto que temperaturas em torno de $20^{\circ} \mathrm{C}$ não resultaram em aumento na quantidade de animais com deformidades. Graham e Hop (1995) também encontraram maior número de deformidades em larvas de bacalhau quando foram expostas a temperaturas mais altas.

Em experimento realizado por Wang e Tsai (2000), observaram que quando larvas de tilápia ( $O$. mossambicus) com zero a cinco dias de idades foram expostas a temperaturas entre 28 a $32^{\circ} \mathrm{C}$, houve aumento na proporção de indivíduos que apresentam deformidades, enquanto que temperaturas em torno de $20^{\circ} \mathrm{C}$ não tiveram influência nessa proporção. Graham e Hop (1995) também encontraram maior número de deformidades em larvas de bacalhau quando foram expostas a temperaturas mais altas. Wang e Tsai (2000) também afirmaram que os mecanismos pelos quais temperaturas elevadas induzem à deformidade de teleósteos ainda não é clara e precisa ser mais investigada. 
Figura 4. Radiografias de alevinos de tilápia com tamanho médio de 51,8 $\pm 11,6 \mathrm{~mm}$ submetidos aos diferentes tratamentos nas temperaturas $28,30,32,34^{\circ} \mathrm{C}$ e do controle (28B).

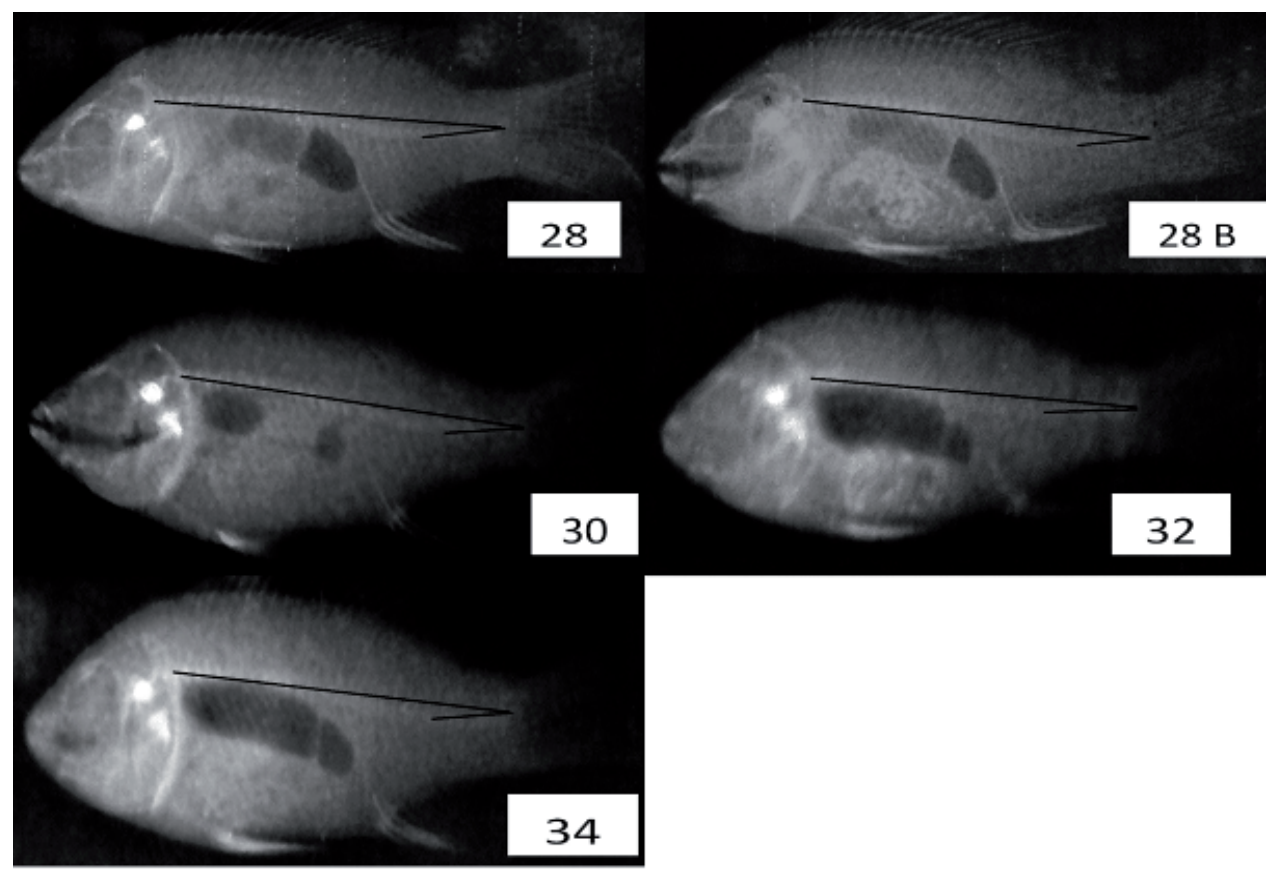

Fonte: Elaboração dos autores.

\section{Conclusão}

Este experimento ficou evidente que as temperaturas de 32 e $34^{\circ} \mathrm{C}$ foram eficientes em promover a inversão sexual de larvas de tilápia do Nilo, sem provocar alterações morfológicas, sugerindo que a temperatura pode ser uma alternativa para a substituição de hormônios durante o processo de inversão sexual.

\section{Referências}

ABUCAY, J. S. C.; MAIR, G. C.; SKIBINSKI, D. O. F.; BEARDMORE, J. A. Environmental sex determination: the effect of temperature and salinity on sex ratio in Oreochromis niloticus L. Aquaculture, Amsterdãn, v. 173, n. 1-4, p. 219-234, 1999.

ALMEIDA, G. S. C. Suplementação dietética de vitamina $c$, desenvolvimento e sanidade do pacu (Piaractus mesopotamicus Holmberg, 1887). 2003. Dissertação (Mestrado em Agronomia)- Escola Superior de Agricultura Luiz de Queiroz. Universidade de São Paulo, Piracicaba.
ANGIENDA, P. O.; AKETCH, B. O.; WAINDI, E. N. Development of All-male fingerlings by heat treatment and the genetic mechanism of heat induced sex determination in nile tilapia (Oreochromis niloticus L.). International Journal of Biological and Life Sciences, Tokio, v 6, n. 1, p. 38-43, 2010.

AZAZA, M. S.; DHRAÏEF, M. N.; KRAÏEM M. M. Effects of water temperature on growth and sex ratio of juvenile Nile tilapia Oreochromis niloticus (Linnaeus) reared in geothermal waters in southern Tunisia. Journal of Thermal Biology, Amsterdãn, v. 33, n. 2, p. 98-105, 2008.

BARAS, E.; JACOBS, B.; MÉLARD, C. Effects of water temperature on survival, growth and phenotypic sex of mixed (XX - XY) progenies of Nile tilapia Oreochromis niloticus. Aquaculture, Amsterdãn, v. 192, n. 2-4, p. $187-$ 199, 2001.

BARAS, E.; MPO'N'TCHA, A.; DRIOUCH, H.; PRIGNON, C.; MÉLARD, C. Ontogenic variations of thermal optimum for growth, and its implication on thermolabile sex determination in blue tilapia. Journal of Fish Biology, Liverpool, v. 61, n. 3, p. 645-660, 2002.

BARAS, E.; PRIGNON, C.; GOHOUNGO, G.; MÉLARD, C. Phenotypic sex differentiation of blue tilapia under constant and fluctuating thermal regimes 
and itsadaptative and evolutionary implications. Journal of Fish Biology, Liverpool, v. 57, n. 1, p. 210-223, 2000.

BAROILLER, J. F.; CHOURROUT, D.; FOSTIER, A.; JALABERT, B. Temperature and sex chromosomes govern the sex ratios of the mouthbreeding cichlid fish Oreochromis niloticus. Journal of Experimental Zoology, Philadelphia, v. 273, n. 3, p. 216-223, 1995.

BEZAULT, E.; FRÉDÉRIC CLOTA, F.; DERIVAZ, M.; CHEVASSUS, B.; BAROILLER, J. F. Sex determination and temperature-induced sex differentiation in three natural populations of Nile tilapia (Oreochromis niloticus) adapted to extreme temperature conditions. Aquaculture, Amsterdãn, v. 272, n. 1, p. S3-S16, 2007. Suplemento.

BORGES, A. M. Piscicultura. 2. ed. Brasília, DF: Emater, 2002.

BORGES, A. M.; MORETTI, J. O. C.; MCMANUS, C.; MARIANTE, A. S. Produção de populações monossexo macho de tilápia-do-nilo da linhagem Chitralada. Pesquisa Agropecuaria Brasileira, Brasília, v. 40, n. 2, p. 153-159, 2005.

BOYD, C. Water quality in ponds for aquaculture. London: Birmingham, 1990.

CARDOSO FILHO, R.; CAMPECHE, D. F. B.; PAULINO, R.V. Tilápia em reservatório de água para irrigação e avaliação da qualidade da água. Revista Brasileira de Ciências Agrárias, Recife, v. 5, n. 1, p. 117122, 2010.

CONOVER, D. O.; KYNARD, B. E. Environmental sex determination: interaction of temperature and genotype in a fish. Science, Washington, v. 213, p. 577-579, 1981.

COSTA, F. T. M.; REIS, F. R. C.; SANTOS, J. M. S.; MACIEL, S. M.; BISERRA, T. S.; MOREIRA, R. L.; FARIAS. W. R. L. Chlorella sp. como suplemento alimentar durante a larvicultura de tilápia do Nilo. Revista Brasileira de Saúde e Produção Animal, Salvador, v. 12, n. 4, p. 1103-1115, 2011.

CURTIS, L. R.; DIREN, F. T.; HURLEY, M. D.; SEIM, W. K.; TUBB, R. A. Disposition and elimination of a methyltestosterone in Nile tilapia, Aquaculture, Amsterdãn, v. 99, n. 1-2, p. 193-201, 1991.

DESPREZ, D.; MÉLARD, C. Effect of ambient water temperature on sex determinism in the blue tilapia Oreochromis aureus. Aquaculture, Amsterdãn, v. 162, n. 1, p. 1-2, 1998.

DIAS-KOBERSTEIN, T. C. R.; NETO, A. G.; STÉFANI, M. V.; MALHEIROS, E. B.; ZANARDI, M. F.; SANTOS, M. A. Reversão sexual de larvas de tilapia do nilo (oreochromis niloticus) por meio de banhos de imersão em diferentes dosagens hormonais. Revista Academica, São José dos Pinhais, v. 5, n. 4, p. 391-395, 2007.

GOLTERMAN, H. L.; CLYMO, R. S.; OHNSTAD, M. A. M. Methodos for physical and chemical analisys of fresh water. Oxford: Blackwell Scientific Publications, 1978.

GRAHAM, M.; HOP, H. Aspects of reproduction and larval biology of arctic cod (Boreogadus saida). Arctic, v. 48 , p. $130-135,1995$.

KARAYÜCEL, I.; PENMAN, D.; KARAYÜCEL, S.; McANDREW, B. Thermal and hormonal feminization of all male YY Nile tilapia, Oreochromis niloticus L. The Israeli Journal of Aquaculture - Bamidgeh, v. 55, n. 2, p. 114-122, 2003.

LIKONGWE, J. S. L.; STECKO, T. D.; JAY, R.; STAUFFER, J. R.; CARLINE, R. F. Combined effects of water temperature and salinity on growth and feed utilization of juvenile Nile tilapia Oreochromis niloticus (Linneaus) Aquaculture, Amsterdãn, v. 146, n. 1, p. 37 46, 1996.

MEURER, F.; HAYASHI, C.; BOSCOLO, W. R.; SCHAMBER, C. R.; BOMBARDELLI, R. A. Fontes protéicas suplementadas com aminoácidos e minerais para tilápia do Nilo durante a reversão sexual, Revista Brasileira de Zootecnia, Viçosa, MG, v. 34, n. 1, p. 1-6, 2005.

PHELPS, R. P.; POPMA, T. J. Sex reversal of tilapia In: COSTA PIERCE, B. A.; RAKOCY, J. E. Tilapia aquaculture in the Americas. Louisiana: World Aquaculture Society, 2000. v. 2, p. 34-59.

POPMA, T. J.; GREEN, B. W. Sex reversal of tilapia in earthen ponds: aquaculture production manual. Alabama: Auburn University, 1990. (Research and development series, 35).

POPMA, T. J.; LOVSHIN, L. Worldwide prospects for commercial production of tilapia. Auburn: Auburn University, 1996. (Research and development, 41).

RINCHARD, J.; DABROWSKI, K.; GARCIAABIADO, M. A.; OTTOBRE, J. Uptake and depletion of plasma 17- $\alpha$-metyltestosterone during induction of masculinization in muskellunge, Esox masquinongy: Effect on plasma steroids and sex-reversal. Steroids, Amsterdãn, v. 64, n. 8, p. 518-525, 1999.

ROTHBARD, S.; ZOHAR, Y.; ZMORA, N.; SIVAN, B. L.; MOAV, B.; YARON, Z. Clearance of 17 $\alpha$-methynyltestosterone from muscle of sex-inversed tilapia hybrids treated for growth enhancement with two doses of the androgen, Aquaculture, Amsterdãn, v. 89, n. 3-4, p. 365-376, 1990. 
ROUGEOT, C.; KANFITINE, S. Y.; PRIGNON, C.; GENNOTTE, V.; MÉLARD, C. Early sex reversal during the embryonic development in the Nile tilapia. Cybium, v. 32, n. 2, p. 104-105, 2008. Suplemento.

SANTANA, F. M. S.; LUCENA, L. B. G.; SANTANA, C. A. S.; SILVA, B. C.; SANTANA, N. M.; MELO, K. S. G. Yield, humidity, acceptance and preference of tilapia submitted to smoking process. Revista Brasileira de Ciências Agrárias, Recife, v. 5, n. 3, p. 423-427, 2010.

SANTOS, E. L.; LUDKE, M. C. M. M.; RAMOS, A. M. P.; BARBOSA, J. M.; LUDKE, J. V.; RABELLO, C. B. V. Digestibilidade de subprodutos da mandioca para a tilápia do Nilo. Revista Brasileira de Ciências Agrárias, Recife, v. 4, n. 3, p. 358-362, 2009.

SCHMITTOU, H. R. High density fish culture in low volume cages. Singapore: American Soybean Association, 1993.

STATISTICAL ANALYSES SYSTEM - SAS. Institute Inc., SAS 9.1.3. Help and Documentation. Cary, NC: SAS Institute Inc., 2002-2004.
TSADIK, G. G.; BART, A. N. Effects of feeding, stocking density and water-flow rate on fecundity, spawning frequency and egg quality of Nile tilapia, Oreochromis niloticus (L.). Aquaculture, Amsterdãn, v. 272, n. 1, p. 380-388, 2007.

WANG, L. H.; TSAI, C. L. Effects of temperature on the deformity and sex differentiation of tilapia, Oreochromis mossambicus. Journal of Experimental Zoology, Philadelphia, v. 286, n. 5, p. 534-537, 2000.

WESSELS, S.; HÖRSTGEN-SCHWARK, G. Selection experiments to increase the proportion of males in Nile tilapia (Oreochromis niloticus) by means of temperature treatment. Aquaculture, Amsterdãn, v. 272, n. 1, S80-S87, 2007. Suplemento.

WESSELS, S.; SAMAVATI, S.; HÖRSTGENSCHWARK, G. Effect of early temperature treatments on sex differentiation in Nile tilapia, Oreochromis niloticus lines selected for high and low thermo-sensitivity. Aquaculture, Amsterdãn, v. 316, n. 1-4, p. 139-142, 2011. 
